PROCEEDINGS OF THE

AMERICAN MATHEMATICAL SOCIETY

Volume 136, Number 10, October 2008, Pages 3675-3681

S 0002-9939(08)09321-0

Article electronically published on May 23, 2008

\title{
A SIMPLE PROOF OF THE MORSE-SARD THEOREM IN SOBOLEV SPACES
}

\author{
ALESSIO FIGALLI
}

(Communicated by David Preiss)

\begin{abstract}
In this paper we give a new simple proof of a result of Luigi De Pascale, which states that the Morse-Sard Theorem holds under the hypothesis of Sobolev regularity. Moreover, as our proof is independent of the MorseSard Theorem with $C^{k}$ regularity, our result implies the classical Morse-Sard Theorem.
\end{abstract}

The Morse-Sard Theorem is concerned with the size of the image of the critical values of a differentiable function. To recall it and to state our result, we need some definitions.

Definition 1. Let $\Omega \subset \mathbb{R}^{n}$ be open and let $f: \Omega \rightarrow \mathbb{R}^{m}$ be a $C^{1}$ function. A point $x \in \Omega$ is said to be a critical point if $D f(x)$ is not of maximum rank. A point $y \in f(\Omega)$ is said to be a critical value if $y=f(x)$ for a critical point $x$. The set of all the critical points is called the critical set.

Let us denote by $\mathscr{L}^{m}$ the $m$-dimensional Lebesgue measure. We can now recall the classical Morse-Sard Theorem (for a proof, see [1, Paragraph 15]):

Theorem 2 (Morse-Sard). Let $\Omega \subset \mathbb{R}^{n}$ be open and let $f: \Omega \rightarrow \mathbb{R}^{m}$ be a $C^{n-m+1}$ function, with $n \geq m\left(C^{1}\right.$ if $\left.m>n\right)$. Then the set of critical values of $f$ has $\mathscr{L}^{m}$-measure zero.

After that theorem, many generalizations have been proved and, at the same time, many counterexamples have been found in the case of not sufficient regularity. In particular, in 2] the same conclusion of the Morse-Sard Theorem has been proved under the only assumption of a $C^{n-m, 1}$ regularity, while in [3] only a $W^{n-m+1, p}$ regularity, with $p>n$, is assumed (see $[3$ for more historical notes). Here we give a simple proof of the result in 3 . We remark that, as our proof is independent of Theorem 2 our result implies the classical Morse-Sard Theorem.

In the proof of our theorem, we will need a refined version of the classical Morrey inequality (for a proof, see [4, paragraph 4.5.3]):

Lemma 3. Let $\Omega \subset \mathbb{R}^{n}$ be an open subset and let $B(x, r)$ be a ball contained in $\Omega$. Then for any $y \in B(x, r)$ we have

$$
|u(x)-u(y)| \leq C r^{1-\frac{n}{p}}\left(\int_{B(x, r)}|D u(z)|^{p} d z\right)^{\frac{1}{p}} \quad \forall u \in W^{1, p} .
$$

Received by the editors April 6, 2006, and, in revised form, June 20, 2006.

2000 Mathematics Subject Classification. Primary 58C25; Secondary 46T20.

Key words and phrases. Sard theorem, Sobolev spaces. (C)2008 American Mathematical Society
Reverts to public domain 28 years from publication 
We remark that, in particular, this inequality gives the embedding $W^{1, p} \hookrightarrow$ $C^{0, \alpha(p)}$, with $\alpha(p)=1-\frac{n}{p}$, and, in more generality, $W^{l, p} \hookrightarrow C^{l-1, \alpha(p)}$.

We will also need the Kneser-Glaeser Rough Composition Theorem. In order to state it, we recall that, given a positive integer $s$, a map $f$ is said to be $s$-flat on $A$ if $D^{j} f(x)=0$ for $j=1, \ldots, s$ for any $x \in A$.

Theorem 4. Let $W \subset \mathbb{R}^{m}$ and $V \subset \mathbb{R}^{n}$ be open sets; $A^{*} \subset W$ and $A \subset V$, with $A$ closed relative to $V ; f: V \rightarrow \mathbb{R}^{p}$ of class $C^{r}$ on $V$ and $s$-flat on $A ; g: W \rightarrow V$ of class $C^{r-s}$ with $g\left(A^{*}\right) \subset A$. Then there is a map $H: W \rightarrow \mathbb{R}^{p}$ of class $C^{r}$ satisfying:

(i) $H(x)=f(g(x))$ for $x \in A^{*}$;

(ii) $H$ is $s$-flat on $A^{*}$.

The proof of this theorem relies on Whitney's Extension Theorem (see for example [1, Theorem 13.2]). Indeed, differentiating the identity $H=f \circ g$, one prescribes the derivative of $H$ on $A^{*}$, and then one only needs to check that the hypotheses needed to apply Whitney's Theorem are satisfied (see [1, Theorem 14.1] for a detailed proof).

Theorem 5. Let $\Omega \subset \mathbb{R}^{n}$ be open and let $f: \Omega \rightarrow \mathbb{R}^{m}$ be a $W_{\text {loc }}^{n-m+1, p}$ function, with $p>n \geq m$. Then the set of critical values of $f$ has $\mathscr{L}^{m}$-measure zero.

Remark. As $W^{n-m+1, p} \hookrightarrow C^{n-m, \alpha(p)}$, we will always refer to the $C^{n-m, \alpha(p)}$ representative. Moreover we observe that with the only assumption of $C^{n-m, \alpha}$ regularity with $\alpha<1$ the result is false. The key point is in fact the existence of another weak derivative summable enough, as we will see in the proof.

Proof. First we observe that, as it suffices obviously to prove the theorem for $f$ restricted to each compact set of $\Omega$, we can assume that $\Omega$ is bounded and that $f \in$ $W^{n-m+1, p}\left(\Omega, \mathbb{R}^{m}\right)$. Thanks to this remark, in the sequel we will always skip the subscript loc.

To simplify the notation, we define $k:=n-m+1$. We remark that, in the case $n=m$, the result is just a corollary of the area formula for Sobolev functions 1 (for a proof and for more references on the subject, see [5]), so we can assume $n>m$, that is, $k \geq 2$.

Let $C_{f}$ be the critical set of $f$ and let us define the sets

$$
A_{s}:=\left\{x \in \Omega \mid D^{i} f(x)=0 \text { for } 1 \leq i \leq s\right\}, \quad 1 \leq s \leq n-m,
$$

and

Then we have

$$
K:=\{x \in \Omega \mid 1 \leq \operatorname{rank} D f(x) \leq m-1\}
$$

$$
C_{f}=K \cup\left(\left(A_{1} \backslash A_{2}\right) \cup\left(A_{2} \backslash A_{3}\right) \cup \ldots \cup\left(A_{n-m-1} \backslash A_{n-m}\right) \cup A_{n-m}\right) .
$$

We will divide the proof into three steps. First we will see that one can always assume that $K=\emptyset$, that is, $C_{f}=\{D f=0\}$. Then, in the second step, we will prove that $\mathscr{L}^{m}\left(f\left(A_{n-m}\right)\right)=0$. This will conclude the proof of the theorem in

\footnotetext{
1 Indeed we will see that, by our proof, one also has the following result: if $f \in W_{l o c}^{1, p}\left(\Omega, \mathbb{R}^{n}\right)$, with $p>n$, and $E$ is a $\mathscr{L}^{n}$-null set, then $\mathscr{L}^{n}(f(E))=0$ (see the first part in the proof of Step 2). This fact, the classical area formula for Lipschitz functions, and a standard approximation of $W^{1, p}$ functions with Lipschitz ones, imply the validity of the area formula also in the Sobolev case.
} 
the case $n=m+1$ (as, in this case, $C_{f}=K \cup A_{1}$ ) and will allow us to start an induction argument on $n-m$. In fact, once we have proved the second step, we can assume that the theorem holds for $W^{n-m, k}$ maps from an open subset of $\mathbb{R}^{n-1}$ to $\mathbb{R}^{m}$. In the third step, thanks to an Implicit Function Theorem, we will reduce the dimension from $n$ to $n-1$ and we will conclude the proof by the inductive hypothesis.

Step 1 (we can assume $K=\emptyset$ ). This is essentially Step 1 in the proof of Theorem 4.1 in 3 .

Let $K_{i}:=\{x \in \Omega \mid \operatorname{rank} D f(x)=i\}, 1 \leq i \leq m-1$, and fix $\bar{x} \in K_{i}$. We can assume $\operatorname{det}\left(\frac{\partial\left(f_{1}, \ldots, f_{i}\right)}{\partial\left(x_{1}, \ldots, x_{i}\right)}\right)(\bar{x}) \neq 0$. Then, in a small relatively compact neighborhood $V$ of $\bar{x}$, we can take as coordinates $\left(y_{1}, \ldots, y_{n}\right)=Y(x):=\left(f_{1}(x), \ldots, f_{i}(x), x_{i+1}, \ldots\right.$, $\left.x_{n}\right)$. So, defining $X:=Y^{-1}, f$ assumes the form

$$
f(X(y))=\left(y_{1}, \ldots, y_{i}, g\left(y_{1}, \ldots, y_{n}\right)\right) .
$$

Now, since $Y \in W^{k, p}(V), D Y$ is invertible, $k \geq 2$ and $p>n$, it is simple to verify that $X$ is still $W^{k, p}$. Moreover, for $k \geq 2$ and $p>n$ the composition of functions in $W_{l o c}^{k, p}$ is still $W_{l o c}^{k, p}$, and so we deduce that $g \in W^{k, p}\left(\tilde{V}, \mathbb{R}^{m-i}\right)$, with $\tilde{V}:=Y(V)$. In these coordinates we have

$$
D(f \circ X)(y)=\left(\begin{array}{cc}
I d_{i} & 0 \\
* & D\left(\left.g\right|_{\left(y_{1}, \ldots, y_{i}\right)}\right)
\end{array}\right)
$$

where $\left.g\right|_{\left(y_{1}, \ldots, y_{i}\right)}: \tilde{V}_{\left(y_{1}, \ldots, y_{i}\right)} \rightarrow \mathbb{R}^{m-i}$ is defined by $\left.g\right|_{\left(y_{1}, \ldots, y_{i}\right)}\left(y_{i+1}, \ldots, y_{n}\right)=g\left(y_{1}\right.$, $\left.\ldots, y_{n}\right)$, with $\tilde{V}_{\left(y_{1}, \ldots, y_{i}\right)}:=\left\{\left(z_{1}, \ldots, z_{n-i}\right) \in \mathbb{R}^{n-i} \mid\left(y_{1}, \ldots, y_{i}, z_{1}, \ldots, z_{n-i}\right) \in \tilde{V}\right\}$. Observing that $\operatorname{rank} D(f \circ X)=\operatorname{rank} D f=i$, thanks to the Slicing Theorem for Sobolev functions (see [4, paragraph 4.9.2]) we have, for $\mathscr{L}^{i}$-a.e. $\left(y_{1}, \ldots, y_{i}\right)$,

$$
\left.g\right|_{\left(y_{1}, \ldots, y_{i}\right)} \in W^{k, p}\left(\tilde{V}_{\left(y_{1}, \ldots, y_{i}\right)}, \mathbb{R}^{m-i}\right)
$$

and

$$
D\left(\left.g\right|_{\left(y_{1}, \ldots, y_{i}\right)}\right)=0 \quad \text { on } \tilde{V}_{\left(y_{1}, \ldots, y_{i}\right)} \cap Y\left(K_{i}\right) .
$$

Once we will have proved the result in the case $K=\emptyset$, applying it to $\left.g\right|_{\left(y_{1}, \ldots, y_{i}\right)}$ we get

$$
\mathscr{L}^{m-i}\left(\left.g\right|_{\left(y_{1}, \ldots, y_{i}\right)}\left(\tilde{V}_{\left(y_{1}, \ldots, y_{i}\right)} \cap Y\left(K_{i}\right)\right)\right)=0 \quad \text { for } \mathscr{L}^{i} \text {-a.e. }\left(y_{1}, \ldots, y_{i}\right)
$$

By Fubini's Theorem, $\mathscr{L}^{m}\left(f\left(V \cap K_{i}\right)\right)=\mathscr{L}^{m}\left(f \circ X\left(\tilde{V} \cap Y\left(K_{i}\right)\right)\right)=0$, and this concludes the proof of the reduction to the case $K=\emptyset$.

Step $2\left(\mathscr{L}^{m}\left(f\left(A_{n-m}\right)\right)=0\right)$. We recall that, by the remark made at the beginning of the proof, we can assume that $\Omega$ is bounded; this implies, in particular, that $A_{n-m}$ has finite Lebesgue measure.

Let $x \in A_{n-m}=A_{k-1}, y \in B(x, r)$ with $r$ such that $B(x, r) \subset \Omega$. As $D^{k-1} f \in$ $W^{1, p}(\Omega)$, by the Taylor formula with integral remainder and by (1) we get

$$
\begin{aligned}
|f(y)-f(x)| & \leq \int_{0}^{1} \frac{(1-t)^{k-2}}{(k-2) !}\left|D^{k-1} f(x+t(y-x))-D^{k-1} f(x)\right||y-x|^{k-1} d t \\
& \leq C r^{k-\frac{n}{p}}\left(\int_{B(x, r)}\left|D^{k} f(z)\right|^{p} d z\right)^{\frac{1}{p}},
\end{aligned}
$$


which implies

$$
|f(y)-f(x)|^{m} \leq C r^{m\left(k-\frac{n}{p}\right)}\left(\int_{B(x, r)}\left|D^{k} f(z)\right|^{p} d z\right)^{\frac{m}{p}} .
$$

Now, using Young's inequality with exponents $\frac{p}{p-m}$ and $\frac{p}{m}$ and taking $r=|y-x|$, we get

$$
|f(y)-f(x)|^{m} \leq C|x-y|^{\frac{p m}{p-m}\left(k-\frac{n}{p}\right)}+C\left(\int_{B(x,|y-x|)}\left|D^{k} f(z)\right|^{p} d z\right) .
$$

As $k=n-m+1$ and $m(k-1) \geq k-1$, we have $k m \geq k+m-1=n$, which implies $\frac{p m}{p-m}\left(k-\frac{n}{p}\right)=\frac{m}{p-m}(p k-n) \geq n$. So, for $|y-x| \leq r \leq 1$ and $x \in A_{k-1}$, we have the estimate

$$
|f(y)-f(x)|^{m} \leq C \int_{B(x, r)}\left(1+\left|D^{k} f(z)\right|^{p}\right) d z .
$$

We now write $A_{n-m}=F_{1} \cup F_{2}$, where

$$
F_{1}:=\left\{\text { density points for } A_{n-m}\right\} \cap\left\{\text { Lebesgue points of }\left|D^{k} f\right|^{p}\right\}
$$

and

$$
F_{2}:=A_{n-m} \backslash F_{1} .
$$

It's a standard result in measure theory that $\mathscr{L}^{n}\left(F_{2}\right)=0$. Let us now show that $\mathscr{L}^{m}\left(f\left(F_{2}\right)\right)=0$.

Fix $\varepsilon>0$ small. $\mathscr{L}^{n}\left(F_{2}\right)$ being 0 , there exists an open set $E_{\varepsilon} \supset F_{2}$ such that $E_{\varepsilon} \subset \Omega$ and $\mathscr{L}^{n}\left(E_{\varepsilon}\right) \leq \varepsilon$. For any $x \in F_{2}$ we take a ball $B_{x}=B\left(x, r_{x}\right)$ such that $B_{x} \subset E_{\varepsilon}$. We now define $\rho_{x}:=\operatorname{diam} f\left(B_{x}\right)$, and we consider the covering of $f\left(F_{2}\right)$ given by $\mathcal{F}=\left\{B\left(f(x), \rho_{x}\right)\right\}_{x \in F_{2}}$. By Vitali's Covering Theorem (see 4, Paragraph 1.5.1]), there exists $\mathcal{G}=\left\{B\left(f\left(x_{i}\right), \rho_{x_{i}}\right)\right\}_{i \in I}$ a finite or countable collection of disjoint balls in $\mathcal{F}$ such that

$$
F_{2} \subset \bigcup_{i \in I} B\left(f\left(x_{i}\right), 5 \rho_{x_{i}}\right) .
$$

By the definition of $\rho_{x_{i}}$ we have

$$
f\left(B_{x_{i}}\right) \subset B\left(f\left(x_{i}\right), \rho_{x_{i}}\right),
$$

which implies that the balls $B_{x_{i}}$ are also disjoint. Therefore, by (3) we get

$$
\begin{aligned}
& \mathscr{L}^{m}\left(f\left(F_{2}\right)\right) \leq 5^{m} \sum_{i \in I} \mathscr{L}^{m}\left(B\left(f\left(x_{i}\right), \rho_{x_{i}}\right)\right)=C_{m} \sum_{i \in I}\left(\operatorname{diam} f\left(B_{x_{i}}\right)\right)^{m} \\
& \leq C \sum_{i \in I} \int_{B_{x_{i}}}\left(1+\left|D^{k} f(z)\right|^{p}\right) d z \leq C \int_{E_{\varepsilon}}\left(1+\left|D^{k} f(z)\right|^{p}\right) d z,
\end{aligned}
$$

where $C_{m}=5^{m} \mathscr{L}^{m}(B(0,1))$. Letting $\varepsilon \rightarrow 0$, since $\mathscr{L}^{n}\left(E_{\varepsilon}\right) \leq \varepsilon$ we obtain $\mathscr{L}^{m}\left(f\left(F_{2}\right)\right)=0$ as wanted.

In order to prove that $\mathscr{L}^{m}(f(F))=0$, we have to show that $\mathscr{L}^{m}\left(f\left(F_{1}\right)\right)=0$. As we do not have that $\mathscr{L}^{n}\left(F_{1}\right)=0$, we see that the inequality (3) does not suffice, but in this case, as $F_{1}$ consists of the density points of $F$, we will get a better estimate for $|f(y)-f(x)|$ when $x, y \in F_{1}$. 
Fix $P \in \mathbb{N}$ large. For any $x \in F_{1}$ there exists $r_{x}>0$ small such that $B\left(x, 2 r_{x}\right) \subset \Omega$ and the following hold:

$$
\begin{gathered}
\frac{\mathscr{L}^{n}\left(B\left(x, r_{x}\right) \cap F_{1}\right)}{\mathscr{L}^{n}\left(B\left(x, r_{x}\right)\right)}=\frac{\mathscr{L}^{n}\left(B\left(x, r_{x}\right) \cap A_{n-m}\right)}{\mathscr{L}^{n}\left(B\left(x, r_{x}\right)\right)} \geq 1-\frac{1}{2(2 P)^{n}}, \\
f_{B\left(x, 2 r_{x}\right)}\left(1+\left|D^{k} f(z)\right|^{p}\right) d z \leq 2\left(1+\left|D^{k} f(x)\right|^{p}\right)
\end{gathered}
$$

and

$$
\frac{1}{2}\left(1+\left|D^{k} f(x)\right|^{p}\right) \leq f_{B\left(x, r_{x}\right) \cap F_{1}}\left(1+\left|D^{k} f(z)\right|^{p}\right) d z
$$

(this can always be done since $x$ is both a Lebesgue point of the integrated function and a density point of $F_{1}$ ). These equations imply a sort of doubling property: if $x \in F_{1}$, then

$$
\begin{aligned}
\int_{B\left(x, 2 r_{x}\right)} & \left(1+\left|D^{k} f(z)\right|^{p}\right) d z \leq 2^{n+1} \mathscr{L}^{n}\left(B\left(x, r_{x}\right)\right)\left(1+\left|D^{k} f(x)\right|^{p}\right) \\
& \leq 2^{n+2} \mathscr{L}^{n}\left(B\left(x, r_{x}\right) \cap F_{1}\right)\left(1+\left|D^{k} f(x)\right|^{p}\right) \\
& \leq 2^{n+3} \int_{B\left(x, r_{x}\right) \cap F_{1}}\left(1+\left|D^{k} f(z)\right|^{p}\right) d z .
\end{aligned}
$$

Moreover, for each $y \in F_{1} \cap B\left(x, r_{x}\right)$, there exist $P+1$ points $\left\{x_{0}, \ldots, x_{P}\right\} \subset F_{1}$, with $x_{0}=y$ and $x_{P}=x$, such that

$$
\left|x_{i}-x_{i-1}\right| \leq \frac{2 r_{x}}{P} \quad \forall 1 \leq i \leq P .
$$

Indeed, first take $y_{1}, \ldots, y_{P-1}$ to be $P-1$ points on the line segment $[y, x]$ such that $\left|y_{i}-y_{i-1}\right|=\frac{|y-x|}{P}$ and then observe that, by (4),$B\left(y_{i}, \frac{r_{x}}{2 P}\right) \cap F_{1}$ is not empty for each $i$, and so it suffices to take a point $x_{i}$ in that set. By this and (2), it follows that

$$
\begin{aligned}
|f(y)-f(x)| & \leq \sum_{i=1}^{P}\left|f\left(x_{i}\right)-f\left(x_{i-1}\right)\right| \\
& \leq C \sum_{i=i}^{P}\left|x_{i}-x_{i-1}\right|^{k-\frac{n}{p}}\left(\int_{B\left(x_{i}, \frac{2 r_{x}}{P}\right)}\left|D^{k} f(z)\right|^{p} d z\right)^{\frac{1}{p}} \\
& \leq C \sum_{i=1}^{P}\left(\frac{2 r_{x}}{P}\right)^{k-\frac{n}{p}}\left(\int_{B\left(x, 2 r_{x}\right)}\left|D^{k} f(z)\right|^{p} d z\right)^{\frac{1}{p}}
\end{aligned}
$$

whenever $y \in B\left(x, r_{x}\right)$. Again using Young's inequality, we get

$$
|f(y)-f(x)|^{m} \leq C P^{m\left(1-k+\frac{n}{p}\right)} \int_{B\left(x, 2 r_{x}\right)}\left(1+\left|D^{k} f(z)\right|^{p}\right) d z \quad \forall y \in B\left(x, r_{x}\right) .
$$

Thus by (5) we obtain that, for all $x \in F_{1}$,

(6) $|f(y)-f(x)|^{m} \leq C P^{m\left(1-k+\frac{n}{p}\right)} \int_{B\left(x, r_{x}\right) \cap F_{1}}\left(1+\left|D^{k} f(z)\right|^{p}\right) d z \quad \forall y \in B\left(x, r_{x}\right)$.

We are now able to prove that $\mathscr{L}^{m}\left(f\left(F_{1}\right)\right)=0$.

For any $x \in F_{1}$ we take the ball $B_{x}=B\left(x, r_{x}\right)$, where $r_{x}$ was defined above. We now define $\rho_{x}:=\operatorname{diam} f\left(B_{x} \cap F_{1}\right)$, and we consider the covering of $f\left(F_{1}\right)$ 
given by $\mathcal{F}=\left\{B\left(f(x), \rho_{x}\right)\right\}_{x \in F_{1}}$. Using again Vitaly's theorem we find $\mathcal{G}=$ $\left\{B\left(f\left(x_{i}\right), \rho_{x_{i}}\right)\right\}_{i \in I}$ a finite or countable collection of disjoint balls in $\mathcal{F}$ such that

$$
F_{1} \subset \bigcup_{i \in I} B\left(f\left(x_{i}\right), 5 \rho_{x_{i}}\right) .
$$

In this case, by the definition of $\rho_{x_{i}}$ we have

$$
f\left(B_{x_{i}} \cap F_{1}\right) \subset B\left(f\left(x_{i}\right), \rho_{x_{i}}\right),
$$

which implies that the sets $B_{x_{i}} \cap F_{1}$ are disjoint. Arguing as for $F_{2}$, thanks to (6) we obtain

$$
\begin{aligned}
\mathscr{L}^{m}\left(f\left(F_{1}\right)\right) & \leq C \sum_{i \in I}\left(\operatorname{diam} f\left(B_{x_{i}} \cap F_{1}\right)\right)^{m} \\
& \leq C P^{m\left(1-k+\frac{n}{p}\right)} \sum_{i \in I} \int_{B_{x_{i}} \cap F_{1}}\left(1+\left|D^{k} f(z)\right|^{p}\right) d z \\
& \leq C P^{m\left(1-k+\frac{n}{p}\right)} \int_{\Omega}\left(1+\left|D^{k} f(z)\right|^{p}\right) d z,
\end{aligned}
$$

and we conclude by letting $P \rightarrow+\infty$, as $k \geq 2>1+\frac{n}{p}$.

Step $3\left(\mathscr{L}^{m}\left(f\left(A_{s-1} \backslash A_{s}\right)\right)=0\right.$, for $\left.2 \leq s \leq k-1\right)$. Fix $\bar{x} \in A_{s-1} \backslash A_{s}$. In order to prove the claim, it suffices to show that there exists an open neighborhood $V$ of $\bar{x}$ such that $\mathscr{L}^{m}\left(f\left(\left(A_{s-1} \backslash A_{s}\right) \cap V\right)\right)=0$. We recall that, by what we already said, our function is $C^{k-1, \alpha(p)}$. Now, as $\bar{x} \in A_{s-1}, f$ is $(s-1)$-flat at $\bar{x}$, but some partial derivative of order $s$ is not zero. Hence we may assume that

$$
\partial_{n} w(\bar{x}) \neq 0, \quad w(\bar{x})=\partial_{i_{1}} \ldots \partial_{i_{s-1}} f(\bar{x})=0 .
$$

We observe that $w \in C^{k-s, \alpha(p)}$, and hence, by the Implicit Function Theorem, there is an open neighborhood $V$ of $\bar{x}$ such that $V \cap\{w=0\}$ is an $(n-1)$-dimensional $C^{k-s, \alpha(p)}$-graph, and so we have $V \cap A_{s-1} \subset g(W)$, where $W \subset \mathbb{R}^{n-1}$ is open and $g: W \rightarrow \mathbb{R}^{n}$ is $C^{k-s, \alpha(p)}$.

Let us now consider the subset $A^{*} \subset W$ defined by $A^{*}:=\left\{x \in W \mid g(x) \in A_{s-1}\right\}$. By Theorem 4 there exists a function $F: W \rightarrow \mathbb{R}^{m}$ of class $C^{k-1}$ such that:

(i) $F(x)=f(g(x))$ for any $x \in A^{*}$;

(ii) $D F(x)=0$ for any $x \in A^{*}$.

Therefore we have $f\left(A_{s-1} \cap V\right) \subset F\left(C_{F} \cap W\right)$, where $C_{F}$ denotes the critical set of $F$. So it suffices to prove that $\mathscr{L}^{m}\left(C_{F} \cap W\right)=0$, and this follows by the induction hypothesis since

$$
F \in C^{k-1}\left(W, \mathbb{R}^{m}\right) \hookrightarrow W_{l o c}^{k-1, p}\left(W, \mathbb{R}^{m}\right) .
$$

\section{ACKNOWLEDGEMENTS}

It's a pleasure to thank Albert Fathi for useful discussions and to gratefully acknowledge the hospitality of the École Normale Supérieure of Lyon, where this paper was written. I also thank David Preiss and the referee of the paper for their helpful comments. 


\section{REFERENCES}

[1] R. Abraham and J. RobBin: Transversal Mappings and Flows. W. A. Benjamin, Inc., New York-Amsterdam, 1967. MR0240836 (39:2181)

[2] S.M. BATES: Toward a precise smoothness hypothesis in Sard's theorem. Proc. Amer. Math. Soc., 117 (1993), no. 1, 279-283. MR1112486 (93c:58028)

[3] L. De Pascale: The Morse-Sard theorem in Sobolev spaces. Indiana Univ. Math. J., 50 (2001), no. 3, 1371-1386. MR1871360 (2002k:58018)

[4] L.C. Evans And R.F. GariePy: Measure Theory and Fine Properties of Functions. Studies in Advanced Mathematics, CRC Press, Boca Raton, FL, 1992. MR1158660 (93f:28001)

[5] J. Maly, D. SWAnson And W.P. Ziemer: The co-area formula for Sobolev mappings. Trans. Amer. Math. Soc., 355 (2003), no. 2, 477-492. MR.1932709(2004a:46037)

Université de Nice Sophia-Antipolis, Laboratoire J.-A. Dieudonné, CNRS UMR 6621, Parc Valrose, 06108 Nice Cedex 02, France

E-mail address: figalli@unice.fr 